\title{
An investigation of thiol/disulfide homeostasis in patients with Behçet's disease
}

Sevinc Can Sandikci', Seda Colak², Ahmet Omma ${ }^{2}$, Mehmet E. Enecik3 Salim Neselioglu4, Ozcan Erel ${ }^{4}$

\begin{abstract}
${ }^{1}$ Ankara Dışkapı Yııdııım Beyazıt Training and Research Hospital, Ankara, Turkey ${ }^{2}$ Ankara Numune Training and Research Hospital, Ankara, Turkey ${ }^{3}$ Mersin City Hospital, Ankara, Turkey

${ }^{4}$ Department of Clinical Biochemistry, Faculty of Medicine, Ankara Yildirim Beyazit University, Ankara, Turkey
\end{abstract}

Submitted: 19 July 2018

Accepted: 28 September 2018

Arch Med Sci 2020; 16 (6): 1353-1359

DOI: https://doi.org/10.5114/aoms.2019.86703

Copyright @ 2019 Termedia \& Banach

\section{Abstract}

Introduction: Behçet's disease (BD) is a relapsing systemic inflammatory disorder. The diagnosis of BD is primarily based on clinical findings. Current biomarkers are not yet sufficient to diagnose and cannot anticipate the course of the disease and response to treatment. The aim of this study was to evaluate the relationship between the thiol-disulfide balance and disease activity and organ involvement in BD.

Material and methods: A hundred fifty patients with BD and 100 age- and gender-matched healthy controls were included in the study. Disease activity was assessed with the BD Current Activity form score. Serum levels of native thiol (NT), total thiol (TT), and disulfide were measured and the disulfide/native thiol, disulfide/total thiol and native thiol/total thiol levels were calculated for the patient and control groups.

Results: Native thiol, total thiol, native thiol/total thiol values of the BD patients were significantly lower than those of the control group. The disulfide/native thiol, disulfide/total thiol values of BD patients were higher compared to the control group and the disulfide value of the BD group was slightly higher compared to the control group. No correlation was determined between thiol levels and disease activity and organ involvement in BD.

Conclusions: In patients with Behcet's disease, the thiol-disulfide homeostasis balance shifted towards disulfide formation due to thiol oxidation. It may be used as a novel marker in BD because it is easy, practical, fully automated and relatively inexpensive.

Key words: thiol, disulfide, Behçet's disease, disease activity.

\section{Introduction}

Behçet's disease (BD) is a relapsing systemic inflammatory disorder of unknown etiology [1]. The diagnosis of BD is primarily based on clinical findings [2]. Common manifestations are oral aphthous ulcers, genital ulcers, skin lesions, and uveitis. Gastrointestinal tract, central nervous system, and vascular system involvement is less common [3]. The pathogenesis of this disease is unknown, but the pathogenic mechanisms have been reported to be immunoregulatory abnormalities [4].

\author{
Corresponding author: \\ Sevinc Can Sandikçi \\ Ankara Dışkapı Yıldırım \\ Beyazıt Training and \\ Research Hospital \\ Ziraat Mahallesi \\ Şehit Ömer Halisdemir Cad. \\ No: 2 \\ 06110 Ankara, Turkey \\ Phone: +90 3125962000 \\ E-mail: scsandikci@gmail.com
}


Quality of life in inflammatory diseases is associated with disease activity. Omma et al. showed that health-related quality of life was impaired compared to the healthy control group in Sjogren syndrome [5]. It is important to detect the disease activity. Many biomarkers have been studied to examine their potential correlations with disease activity and therapy. However, they are not routinely used in clinical practice as they are not simple or easily derived $[6,7]$. One of them is leptin, which has structural similarity to interleukin 6 (IL-6), IL-12, IL-15, granulocyte colony-stimulating factor (G-CSF). Dervišević et al. reported that serum leptin levels are increased and significantly associated with disease activity in patients with RA [8].

Oxidative stress plays a role in the pathophysiology of Behçet's syndrome. Serum antioxidative status decreases in patients with BD [9]. Previous studies have shown that oxidant radicals increase secondarily to inflammation and autoimmunity in rheumatic diseases [10, 11].

Thiols are sulfur-containing compounds which are functional groups present in the structure of main proteins, and are an important part of the antioxidant cascade. Reactive oxygen radicals are formed because of increased oxidative stress in the environment. Disulfide bonds form following this oxidation. At the first stage of oxidative damage, the oxidant radicals cause oxidation of the thiol groups of sulfur-containing amino acids (cysteine) of proteins and form disulfide (-S-S-) bonds [12]. The resulting disulfide bonds are reduced to thiol groups and the thiol reserves again increase $[13,14]$. Thiols react with reactive oxygen radicals and become oxidized, and remove these radicals to prevent tissue damage. Through these reactions at the cellular level, dynamic thiol/disulfide homeostatic status is maintained [15]. The measurement of thiol-disulfide levels indirectly shows oxidative stress levels.

In 2014, Erel and Neselioglu developed a new spectrophotometric method with high accuracy and sensitivity for the measurement of thioldisulfide [16]. After determination of the native thiol, total thiol and disulfide levels, percentages such as disulfide/native thiol, disulfide/total thiol, and native thiol/total thiol were calculated. Therefore, abnormal thiol/disulfide homeostasis is expected to be related to the etiopathogenesis of different diseases, and a number of studies have demonstrated the significant role of abnormal thiol/disulfide homeostasis in chronic kidney disease, diabetes mellitus, cardiovascular diseases and cancer [17-20].

In this study, it was aimed to measure thiol/disulfide levels and evaluate whether thiol/disulfide imbalance is related to disease activity and organ involvement in $\mathrm{BD}$.

\section{Material and methods}

\section{Study population}

This cross-sectional study included 150 patients with $\mathrm{BD}$ and 100 age- and gender-matched healthy control subjects. The control group consisted of healthy individuals who had been admitted to our hospital's Internal Medicine and Rheumatology clinics. Patients and controls with known concomitant autoimmune or autoinflammatory disease, acute or chronic infection, malignancy, diabetes mellitus, kidney failure, liver disease, thyroid disease, cardiovascular and cerebrovascular disease, pregnancy or postpartum 6 months, or alcohol consumption were excluded from the study.

The diagnosis of BD was made according to the criteria from the International Study Group for BD [21]. Demographic features and clinical characteristics were recorded. Disease activity was assessed with the Turkish version of the Behçet's Disease Current Activity Form (BDCAF) [22]. Behçet's Disease Current Activity Form, which evaluates clinical features present during the last 4 weeks prior to the date of assessment, has been shown to have good interobserver reliability for assessing general disease activity [23]. The items of this activity form are as follows: headache, oral ulcers, genital ulcers, erythema, skin pustules, arthralgia, arthritis, intestinal involvement, new eye involvement, new nervous system involvement, and new major vessel involvement. The BDCAF score was calculated by adding up the scores for each item and ranged from 0 to 12 . And there are two questions which assess disease activity from the patient's and clinician's perspective. These items are a Likert scale, ranging from very bad to very good, to indicate how the patient or the clinician felt the disease had been over the past 4 weeks.

The study protocol was approved by the Local Ethics Committee. The research protocol complies with the 2000 Declaration of Helsinki and written informed consent was obtained from all participants.

\section{Blood sampling}

Venous blood samples were obtained after 12-hour fasting. 10-ml plain tubes and 2-ml vacuum tubes containing ethylenediaminetetraacetic acid (EDTA) were used. Serum samples were separated after centrifugation at $1500 \mathrm{rpm}$ for $10 \mathrm{~min}$ and stored at $-80^{\circ} \mathrm{C}$ until analysis. Native thiol, total thiol and disulfide amounts of the BD and control groups were examined in the same session with the same serum samples. Erythrocyte sedimentation rates (ESR) and C-reactive protein (CRP) levels were measured within $3 \mathrm{~h}$ of obtaining the samples. 


\section{Biochemical analysis}

Serum native thiol (NT), total thiol (TT), and disulfide levels $(\mu \mathrm{mol} / \mathrm{l})$ were measured with the method newly developed by Erel and Neselioglu [16]. In summary, reducible disulfide bonds were first reduced to form free functional thiol groups. Formaldehyde was used to remove unused and consumed sodium borohydride. All of the thiol groups, including reduced and native thiol groups, were determined after reaction with DTNB (5,5 -dithiobis-(2-nitrobenzoic acid)). Half of the difference between total thiol (TT) and native thiols (NT) provided the dynamic disulfide amount (-S-S). After the native thiol, total thiol and disulfide amounts were determined, the percentages of disulfide/total thiol, native thiol/total thiol and disulfide/native thiol were calculated.

For CRP, the immunoturbidimetric method with the Beckman Coulter AU5800 Clinical Chemistry System (normal value: $<5 \mathrm{mg} / \mathrm{l}$ ) was used. The Alifax ESR Analyser System Modified Western Blot Green method (standard value: < $20 \mathrm{~mm} / \mathrm{h}$ ) was used to analyze samples for ESR measurements.

\section{Statistical analysis}

All statistical analyses were conducted using SPSS software (version 18, IBM, Chicago, IL, USA). The Shapiro-Wilk test was used to detect whether the distribution of groups was homogeneous. Continuous variables with normal distribution were presented as mean \pm standard deviation (SD), while continuous variables without normal distribution were presented as median (minimummaximum). Categorical variables were shown as numbers and percentages. The groups were compared using Student's $t$ test for continuous variables with normal distribution and the MannWhitney $U$ test for continuous variables with nonnormal distribution. Categorical variables were analyzed using the $\chi^{2}$ test. Correlation analysis was applied to numeric parameters using Pearson's and Spearman's correlation test. A value of $p<0.05$ was considered statistically significant.

\section{Results}

The BD patients and control group were similar in terms of age (37.49 \pm 9.57 years vs. 38.49 \pm 11.63 years, $p>0.05)$ and gender distribution (M/F; $85 / 65$ vs. $57 / 43, p>0.05)$. The clinical manifestations of the patients are shown in Table I. Of the patient group, 38\% were diagnosed in rheumatology, 34.7\% in dermatology, 9.3\% in internal medicine, $9.3 \%$ in ophthalmology and $8.6 \%$ in other departments. Oral ulcer was observed in $100 \%$ of the patients, genital ulcer in $86 \%$ of patients and $34 \%$ of patients demonstrated positive pathergy tests (erythema nodosum in $49.3 \%$, pap- ulopustular lesion in 65.3\%). Active disease was determined in 114 (76\%) patients and 36 (24\%) patients had inactive disease. Vascular involvement was observed in 50 (33.3\%) patients and ocular involvement in 50 (33.3\%) patients. The mean duration of BD involvement was $8.44 \pm 6.88$ years and the disease duration ranged between 6 months and 38.3 years $(8.44 \pm 6.88)$.

The NT $(357.93 \pm 76.65$ vs. $453.01 \pm 60.8 \mu \mathrm{mol} / \mathrm{l}$, $p<0.001)$, TT (398.57 \pm 78.33 vs. $490.29 \pm 58.48$ $\mu \mathrm{mol} / \mathrm{l}, p<0.001)$, and NT/TT $(p<0.001)$ values of

Table I. Demographic and clinical characteristics of patients with Behçet's disease

\begin{tabular}{|c|c|}
\hline Parameter & Value \\
\hline Gender (F/M) & $\begin{array}{c}65 / 85 \\
(43.3 / 56.7)\end{array}$ \\
\hline Age, mean \pm SD (range) [years] & $\begin{array}{c}37.49 \pm 9.57 \\
(18-64)\end{array}$ \\
\hline $\begin{array}{l}\text { Age at time of diagnosis, mean } \pm \text { SD } \\
\text { (range) [years] }\end{array}$ & $\begin{array}{c}28.89 \pm 8.44 \\
(14-55)\end{array}$ \\
\hline $\begin{array}{l}\text { Disease duration, mean } \pm \text { SD (range) } \\
\text { [years] }\end{array}$ & $\begin{array}{c}8.44 \pm 6.88 \\
(0.08-38.33)\end{array}$ \\
\hline Active disease, $n(\%)$ & $114(76.0)$ \\
\hline BDCAF, median (IQR) & $2.71 \pm 1.65$ \\
\hline Patient's perception of disease activity & $2.21 \pm 1.76$ \\
\hline Clinician's perception of disease activity & $1.41 \pm 1.45$ \\
\hline \multicolumn{2}{|l|}{$\begin{array}{l}\text { Disease manifestations ever presented } \\
\text { by BD patients, } n(\%) \text { : }\end{array}$} \\
\hline Oral ulcers & $150(100.0)$ \\
\hline Genital ulcers & $129(86.0)$ \\
\hline Erythema nodosum & $74(49.3)$ \\
\hline Papulopustular lesions & $98(65.3)$ \\
\hline Positivity of pathergy test & $51(34.0)$ \\
\hline Arthritis & $62(41.3)$ \\
\hline Uveitis & $50(33.3)$ \\
\hline Vascular involvement & $59(33.3)$ \\
\hline Gastrointestinal system involvement & $4(2.7)$ \\
\hline Central nervous system involvement & $9(6.0)$ \\
\hline Pulmonary aneurism & $2(1.3)$ \\
\hline \multicolumn{2}{|l|}{ Medical therapy, $n(\%)$ : } \\
\hline Colchicine & $113(75.3)$ \\
\hline Azathioprine & $70(46.6)$ \\
\hline Cyclophosphamide & $5(3.3)$ \\
\hline Corticosteroids & $43(28.7)$ \\
\hline Interferon & $1(0.7)$ \\
\hline Anti-TNF & $1(0.7)$ \\
\hline
\end{tabular}


Table II. Laboratory results of the Behçet's disease and the control group

\begin{tabular}{|lccc|}
\hline Parameter & BD & HC & $P$-value \\
\hline Native thiol $(\mathrm{NT})[\mu \mathrm{mol} / \mathrm{l}]$ & $357.93 \pm 76.65$ & $453.01 \pm 60.8$ & $<0.001^{*}$ \\
\hline Total thiol $(\mathrm{TT})[\mu \mathrm{mol} / \mathrm{l}]$ & $398.57 \pm 78.33$ & $490.29 \pm 58.48$ & $<0.001^{*}$ \\
\hline Disulfide $[\mu \mathrm{mol} / \mathrm{l}](\mathrm{SS})$ & $20.32 \pm 8.19$ & $18.64 \pm 7.87$ & 0.109 \\
\hline Disulfide/native thiol $\times 100$ & $5.93 \pm 2.76$ & $4.25 \pm 1.99$ & $<0.001^{*}$ \\
\hline Disulfide/total thiol $\times 100$ & $5.2 \pm 2.13$ & $3.86 \pm 1.69$ & $<0.001^{*}$ \\
\hline Native thiol/total thiol $\times 100$ & $89.6 \pm 4.26$ & $92.28 \pm 3.38$ & $<0.001^{*}$ \\
\hline
\end{tabular}

${ }^{* *} p<0.05$. All values were expressed as mean $\pm S D$. Calculated using Student's t-test for normal distribution.

Table III. Correlation between clinical parameters and thiol disulfide parameters in 150 patients with Behçet's disease

\begin{tabular}{|lcccccc|}
\hline Parameter & $\begin{array}{c}\text { Native thiol } \\
(\mathrm{NT})\end{array}$ & $\begin{array}{c}\text { Total thiol } \\
(\mathrm{TT})\end{array}$ & $\begin{array}{c}\text { Disulfide } \\
(\mathrm{SS})\end{array}$ & \% SS/NT & \% SS/TT & \% NT/TT \\
\hline Age & $-0.253^{* *}$ & $-0.252^{* *}$ & 0.020 & $\mathbf{0 . 1 2 6 ^ { * }}$ & $\mathbf{0 . 1 2 7 ^ { * }}$ & $-0.127^{*}$ \\
\hline First symptom age & $-0.310^{* *}$ & $-0.307^{* *}$ & -0.019 & 0.124 & 0.121 & -0.121 \\
\hline Diagnosed age & $-0.301^{* *}$ & $-0.303^{* *}$ & -0.038 & 0.101 & 0.100 & -0.100 \\
\hline Duration of disease & $-0.204^{*}$ & $-0.203^{*}$ & -0.013 & 0.089 & 0.088 & -0.088 \\
\hline BDCAF & -0.080 & -0.104 & -0.124 & -0.099 & -0.098 & 0.098 \\
\hline CRP & -0.145 & $-0.176^{*}$ & $-0.164^{*}$ & -0.092 & -0.094 & 0.094 \\
\hline Sedimentation & -0.053 & -0.104 & $-0.251^{* *}$ & -0.150 & -0.158 & 0.158 \\
\hline
\end{tabular}

${ }^{\star} p<0.05,{ }^{* *} p<0.01$. CRP - C-reactive protein, BDCAF - Behçet's Disease Current Activity Form.

the BD patients were significantly lower compared to the control group. The disulfide/NT $(p<0.001)$, disulfide/TT $(p<0.001)$, CRP $(p<0.001)$ and ESR $(p=0.021)$ values of the BD patients were significantly higher than those of the control group. The disulfide value of the BD group was slightly higher compared to the control group. The thiol and disulfide values of the BD and control groups are presented in Table II.

There was no significant difference between active and inactive patients in the thiol and disulfide values $(p>0.05)$. No difference was determined between the uveitis-positive and uveitis-negative patients or between the vascular involvement positive and negative patients in thiol values $(p>0.05)$.

Correlation analysis revealed a negative correlation between NT levels and demographic and clinical parameters such as age $(r=-0.253, p<0.001)$, first symptom age $(r=-0.310, p<0.001)$, BD diagnosis age $(r=-0.301, p<0.001)$, and disease duration $(r=-0.204, p<0.05)$. There was a negative correlation between TT and age $(r=-0.252, p<0.001)$, first symptom age $(r=-0.307, p<0.001)$, BD diagnosis age $(r=-0.303, p<0.001)$, and CRP $(r=-0.176$, $p<0.05)$. There was a negative correlation between disulfide and CRP $(r=-0.164, p<0.05)$ and
ESR ( $r=-0.251, p<0.001)$. BDCAF was positively correlated with CRP $(r=0.266, p<0.001)$ and ESR $(r=0.219, p<0.001)$ and did not correlate with thiol and disulfide values. The correlation analyses between the parameters in patients with Behcet's disease are given in Table III.

\section{Discussion}

Behçet's disease (BD) is a systemic vasculitis of unknown etiology. Oxidative stress is an important factor in vascular injury. Stimulated neutrophils produce oxygen intermediates, inducing auto-oxidative damage such as tissue injury $[24,25]$. Moreover, as the inflammatory processes are known to increase oxidative stress, activated neutrophils could be the main source of oxidative stress [26]. Niwa et al. suggested that tissue injury in Behcet's disease may be related to the extreme generation of oxygen intermediates (OI) by stimulated polymorphonuclear cells. In patients with the complete type of the disease and in the active stage, markedly increased $\mathrm{OI}$ production has been shown to be present [27]. As shown previously, plasma thiol levels primarily target hypochlorous acid $(\mathrm{HOCl})$ and are the most important component of the plasma antioxidant system [28]. 
Thiol groups are sulfur-containing protein compounds with antioxidant properties, which exist in plasma most prominently as bound to albumin and amino acids. Thiols (RSH) can undergo an oxidation reaction via oxidants and form disulfide (RSSR) bonds [29]. Disulfide bonds are oxidized forms. A disulfide bond is a covalent bond; the linkage is also called an SS bond or disulfide bridge. The disulfide bonds formed can again be reduced to thiol groups. This balance is essential for antioxidant defense and apoptosis. If there is an imbalance, it can result in the formation of reactive oxygen radicals leading to endothelial dysfunction and apoptosis [30, 31]. It has been observed that plasma SH groups act as an important extracellular scavenger of peroxides and are also helpful in protecting the surrounding tissues [32]. Critical SH groups on cell membranes protect proteins against oxidative damage by MPO-derived $\mathrm{HOCl}$, while they are consumed [33]. Dynamic thiol disulfide homeostasis is being increasingly implicated in many disorders, including hypertension, diabetes mellitus, stroke, hypothyroidism, rheumatoid arthritis, and familial Mediterranean fever [34-38]. Kose et al. reported that decreased plasma SH levels were observed in $\mathrm{BD}$ patients, and the decreases in $\mathrm{SH}$ levels indicate that plasma antioxidant defense mechanisms may be impaired and insufficient in BD [39].

It is complicated whether this thiol/disulfide homeostasis is primary or secondary to the disease process. Thiol/disulfide homeostasis was impaired. Thiol levels were lower than in the healthy control group; therefore, these patients might have been exposed to oxidative stress. Oxidative stress plays an important role in endothelial dysfunction in BD. Our findings suggest that the deficiency of thiol/disulfide homeostasis may play a role in the pathogenesis of BD. So thiol/ disulfide homeostasis is one of the primary causes of the disease process. On the other hand, chronic inflammation might be the major cause of increase in oxide thiol form. Therefore, changes in thiol/disulfide balance caused by oxidative stress showed that it is secondary to the disease process. This is a cross sectional study; we cannot say whether it is primary or secondary to the disease process.

The measurement of dynamic thiol/disulfide was first started with a fully automated, simple and cost efficient method developed by Erel et al. [16]. Thiol and disulfide in low molecular weight compounds of cystine, reduced glutathione (GSH) and oxidized glutathione (GSSG) measured by the old method do not reflect the exact thiol/disulfide status of the body. However, the new method can measure a large portion of the thiols (albumin and other proteins) [40-42].

In the current study, lower levels of NT and TT were determined in $\mathrm{BD}$ patients compared to the healthy control group. The disulfide levels of BD patients were also slightly higher than those of the healthy control subjects. Isik et al. observed that there was no significant difference between active and inactive patient groups with respect to oxidants and antioxidants and the serum SH level was lower in the BD group than the healthy group, as was seen in the current study [9]. Yazici et al. suggested that there may be inflammation leading to oxidative stress, especially protein oxidation, not only in patients with active BD but also in those with inactive BD who have decreased thiol levels [28].

According to the results of the current study, thiol groups are severely diminished in BD patients compared to healthy control subjects. Therefore, $\mathrm{BD}$ patients may experience severe oxidative stress damage.

A negative correlation was found in the current study between age and thiol. Therefore, with the results of this analysis, it can be said that thiol levels decrease with increasing age, while disulfide levels increase. Previous studies have also demonstrated that oxidative stress increases with age, and as a result, a thiol/disulfide homeostatic imbalance occurs $[16,43]$. Correlation analysis showed that first symptom age, diagnosis age and disease duration were negatively correlated with native thiol and total thiol levels. There was a negative correlation between ESR and CRP and disulfide level and a positive correlation between ESR and CRP and patients' perception, clinicians' perception of disease activity and BDCAF. Bekpinar et al. reported a decrease in the $\mathrm{SH}$ level in $\mathrm{BD}$ and no correlation between the severity of $\mathrm{BD}$ and the measured parameters [44].

The main limitation of this study was the crosssectional design. In addition, this study gives no clues about the actual relationship between the alteration in serum thiol levels and changes in disease activity or specific organ involvement in BD. All patients were already taking corticosteroids and immunosuppressive agents, which might have affected thiol levels when the blood samples were collected. The other limitation of this study was that we could not correlate the results of thiol levels with ongoing laboratory measurement such as neutrophil/lymphocyte or monocyte/lymphocyte ratios. However, this study can be accepted as a large study as it focuses on thiol as a different biomarker of oxidative stress in patients with BD.

In conclusion, the finding that dynamic thiol/ disulfide homeostasis shifts towards disulfide formation as a result of increased oxidative stress provides a novel insight into the mechanisms underlying BD. These results confirm the presence of oxidative stress in patients with BD and show the need for further prospective and large-scale studies on this subject. 


\section{Acknowledgments}

The authors thank the staff of Ankara Numune Training and Research Hospital, Department of Rheumatology for their generous friendly assistance at every step of this study.

\section{Conflict of interest}

The authors declare no conflict of interest.

\section{References}

1. Gul A. Behçet's disease: an update on the pathogenesis. Clin Exp Rheumatol 2001; 19 (5 Suppl 24): S6-12.

2. Alpsoy E. Behcet's disease: a comprehensive review with a focus on epidemiology, etiology and clinical features, and management of mucocutaneous lesions. J Dermatol 2016; 43: 620-32.

3. El-Ageb EM, Al-Maini MH, Al-Shukaily AK, Al-Farsi Y, Richens ER. Clinical features of Behçet's disease in patients in the Sultanate of Oman; the significance of the antiphospholipid antibodies? Rheumatol Int 2002; 21: 176-81.

4. Watanabe K, Ohashi Y. Natural killer cell activity in patients with Behcet's Disease. Am J Ophthalmol 1984; 98: 813-4.

5. Omma A, Tecer D, Kucuksahin O, Sandikci SC, Yildiz F, Erten S. Do the European League Against Rheumatism (EULAR) Sjögren's syndrome outcome measures correlate with impaired quality of life, fatigue, anxiety and depression in primary Sjögren's syndrome? Arch Med Sci 2018; 14: 830-7.

6. Cantarini L, Pucino V, Vitale A, et al. Immunometabolic biomarkers of inflammation in Behcet's disease: relationship with epidemiological profile, disease activity and therapeutic regimens. Clin Exp Immunol 2016; 184: 197-207.

7. Jiang Y, Zang M, Li S. Serum PLR and LMR in Behcet's disease: can they show the disease activity? Medicine (Baltimore) 2017; 96: e6981.

8. Dervišević A, Resić H, Sokolović Š, et al. Leptin is associated with disease activity but not with anthropometric indices in rheumatoid arthritis patients. Arch Med Sci 2018; 14: 1080-6.

9. Isik A, Koca SS, Ustundag B, Selek S. Decreased total antioxidant response and increased oxidative stress in Behcet's disease. Tohoku J Exp Med 2007; 212: 133-41.

10. Ozyazgan S, Andican G, Erman H, et al. Relation of protein oxidation parameters and disease activity in patients with Behçet's disease. Clin Lab 2013; 59: 819-25.

11. Acikgoz N, Ermiş N, Yağmur J, et al. Elevated oxidative stress markers and its relationship with endothelial dysfunction in Behçet disease. Angiology 2011; 62: 296-300.

12. Finkel T. Redox-dependent signal transduction. FEBS Lett 2000; 476: 52-4.

13. Jones DP, Liang Y. Measuring the poise of thiol/disulfide couples in vivo. Free Radic Biol Med 2009; 47: 1329-38.

14. Şahin A, Erten Ş, Altunoğlu A, et al. Comparison of serum oxidant and antioxidant parameters in familial Mediterranean fever patients (FMF) with attack free period. Acta Reumatol Port 2014; 39: 316-21.

15. Ates I, Kaplan M, Inan B, et al. How does thiol/disulfide homeostasis change in prediabetic patients? Diabetes Res Clin Pract 2015; 110: 166-71.
16. Erel O, Neselioglu S. A novel and automated assay for thiol/disulphide homeostasis. Clin Biochem 2014; 47: 326-32.

17. Rodrigues SD, Batista GB, Ingberman M, Pecoits-Filho R, Nakao LS. Plasma cysteine/cystine reduction potential correlates with plasma creatinine levels in chronic kidney disease. Blood Purif 2012; 34: 231-7.

18. Matteucci E, Giampietro O. Thiol signalling network with an eye to diabetes. Molecules 2010; 15: 8890-903.

19. Go YM, Jones DP. Cysteine/cystine redox signaling in cardiovascular disease. Free Radic Biol Med 2011; 50: 495-509.

20. Prabhu A, Sarcar B, Kahali S, et al. Cysteine catabolism: a novel metabolic pathway contributing to glioblastoma growth. Cancer Res 2014; 74: 787-96.

21. International Study Group for Behcet's Disease. Criteria for diagnosis of Behcet's disease. Lancet 1990; 335: 1078-80.

22. Hamuryudan V, Fresko I, Direskeneli $\mathrm{H}$, et al. Evaluation of the Turkish translation of a disease activity form for Behcet's syndrome. Rheumatology (Oxford) 1999; 38: 734-6.

23. Bhakta BB, Brennan P, James TE, Chamberlain MA, Noble BA, Silman AJ. Behcet's disease: evaluation of a new instrument to measure clinical activity. Rheumatology (Oxford) 1999; 38: 728-33.

24. Fridovich I. Oxygen radicals, hydrogen peroxide and oxygen toxicity. In: Free radicals in biology. Pryor WA (ed.). Academic Press, New York 1976; 239-77.

25. Sacks T, Moldow CF, Craddock PR, Bowers TK, Jacob HS. Oxygen radicals mediate endothelial cell damage by complement-stimulated granulocytes. An in vitro model of immune vascular damage. J Clin Invest 1978; 61: 1161-7.

26. Sies H. Oxidative stress: oxidants and antioxidants. Exp Physiol 1997; 82: 291-5.

27. Niwa Y, Miyake S, Sakane T, Shingu M, Yokoyama M. Auto-oxidative damage in Behcet's disease endothelial cell damage following the elevated oxygen radicals generated by stimulated neutrophils. Clin Exp Immunol 1982; 49: 247-55.

28. Yazici C, Kose K, Caliş M, Demlr M, Kirnap M, Ateş F. Increased advanced oxidation protein products in Behcet's disease: a new activity marker? Br J Dermatol 2004; 151: 105-11.

29. Cremers CM, Jakob U. Oxidant sensing by reversible disulfide bond formation. J Biol Chem 2013; 288: 26489-96.

30. Biswas S, Chida AS, Rahman I. Redox modifications of protein-thiols: emerging roles in cell signaling. Biochem Pharmacol 2006; 71: 551-64.

31. Circu ML, Aw TY. Reactive oxygen species, cellular redox systems, and apoptosis. Free Radic Biol Med 2010; 48: 749-62.

32. Hall ND, Maslen CL, Blake DR. The oxidation of serumsulph-hydryl groups by hydrogen peroxide secreted by stimulated phagocytic cells in rheumtoid arthritis. Rheumatol Int 1984; 4: 35-8.

33. Weiss SJ. Tissue destruction by neutrophils. N Eng J Med 1989; 320: 365-76

34. Ates I, Ozkayar N, Inan B, et al. Dynamic thiol/disulphide homeostasis in patients with newly diagnosed primary hypertension. J Am Soc Hypertens 2016; 10: 159-66.

35. Kundi $H$, Ates I, Kiziltunc E, et al. A novel oxidative stress marker in acute myocardial infarction; thiol/disulphide homeostasis. Am J Emerg Med 2015; 33: 1567-71.

36. Gao C, Li T, Liu J, Guo Q, Tian L. Endothelial functioning and hemodynamic parameters in rats with subclinical hypothyroid and the effects of thyroxine replacement. PLoS One 2015; 10: e0131776.

37. Tetik S, Ahmad S, Alturfan AA, et al. Determination of oxidant stress in plasma of rheumatoid arthritis and pri- 
mary osteoarthritis patients. Indian J Biochem Biophys 2010; 47: 353-8.

38. Omma A, Sandikci SC, Kücüksahin O, Alisik M, Erel O. Can the thiol/disulfide imbalance be a predictor of colchicine resistance in familial mediterranean fever? J Korean Med Sci 2017; 32: 1588-94.

39. Kose K, Dogan P, Ascioglu M, Erkilic K, Ascioglu O. Oxidative stress and antioxidant defenses in plasma of patients with Behcet's disease. Tohoku J Exp Med 1995; 176: 239-48.

40. Glowacki R, Bald E. Fully automated method for simultaneous determination of total cysteine, cysteinylglycine, glutathione and homocysteine in plasma by HPLC with UV absorbance detection. J Chromatogr B Analyt Technol Biomed Life Sci 2009; 877: 3400-4.

41. Chen W, Zhao Y, Seefeldt T, Guan X. Determination of thiols and disulfides via HPLC quantification of 5-thio-2-nitrobenzoic acid. J Pharm Biomed Anal 2008; 48: 1375-80.

42. Turell L, Radi R, Alvarez B. The thiol pool in human plasma: the central contribution of albumin to redox processes. Free Radic Biol Med 2013; 65: 244-53.

43. Kirlin WG, Cai J, Thompson SA, Diaz D, Kavanagh TJ, Jones DP. Glutathione redox potential in response to differentiation and enzyme inducers. Free Radic Biol Med 1999; 27: 1208-18.

44. Bekpinar S, Kiliç N, Unlucerci Y, Köse AA, Azizlerli G, Kirt ZO. Evaluation of nitrosative and oxidative stress in Behçet disease. J Eur Acad Dermatol Venereol 2005; 19: 167-71. 